\title{
The Oral Mucosa: Subtypes of Epidermolysis Bullosa
}

\author{
Michel Goldberg ${ }^{1 *}$ \\ ${ }^{1}$ Department of Oral Biology. Faculty of Fundamental and Biomedical Sciences, Paris Cité University, France.
}

*Corresponding Author: Michel Goldberg, Department of Oral Biology. Faculty of Fundamental and Biomedical Sciences, Paris Cité University, France; Tel: +33-1662676709; Email: mgoldod@ gmail.com

Received Date: 30-04-2020; Accepted Date: 08-05-2020; Published Date: 15-05-2020

Copyright $^{\oplus} 2020$ by Goldberg M. All rights reserved. This is an open access article distributed under the terms of the Creative Commons Attribution License, which permits unrestricted use, distribution, and reproduction in any medium, provided the original author and source are credited.

\begin{abstract}
The oral cavity, which consists of the lips, vestibule, gums, hard and soft palates, tongue, tonsil, uvula and salivary glands, is covered by a superficial pluristratified epithelium. The oral mucosa includes masticatory, lining, or specialized layers. The basement membrane separates the epithelium from the mucosa. Subtypes of have been identified: Epidermolysis Bullosa Aquisita (EBA), Epidermolysis Bullosa Simplex (EBS), junctional, and dystrophic. Blisters are characterised in this genetic pathology as epidermolysis bullosa simplex (suprabasal), and Epidermolysis Bullosa Junctionalis (EBJ) (located in the different layers of the basement membrane). This form have distinct character by separation within epithelial keratinocytes, or between the epithelial cells and the basement membrane (Herlitz form, involving both the skin and the lamina propria, blistering occurring at sites of friction). Dystrophic Epidermolysis Bullosa (DEB) involves discontinuities in the underlying connective tissue. There is no efficient therapy for EB, resulting from genetic mutations. Treatment helps to control symptoms but not treat these disorders.
\end{abstract}

\section{Keywords}

Epidermolysis Bullosa; Keratin Mutations; Basement Membrane; Blistering; Laminin; Collagen Mutations 


\section{Introduction}

\section{The Composition and Structure of the Oral Mucosa}

The oral mucosa is made up of different types epithelial structures across the oral cavity, including the inner face of the cheeks, the palate and the floor of the mouth [1]. The free and attached gingiva is made of stratified non-keratinized epithelium. In contrast, along the hard palate, the dorsal surface of the tongue and around the emergence of teeth is keratinized. Keratinized and non-keratinized epithelia share the totality of the oral mucosa.

The oral mucosa comprises a specific number of domains including:

(1) The masticatory mucosa (gingiva and hard plate), subjected to mechanical forces of mastication. This epithelium is keratinized and very similar to the epidermis of skin. It is attached to the underlining structures by a dense collagenous connective tissue.

(2) The lining mucosa is cover all the remaining regions except the dorsal surface of the tongue. It is covered by a variable thickness of non-keratinized epithelium that is with a variable thickness.

(3) The specialized mucosa covers the tongue and display different type of papillae: filiform, foliate, circumvallate/goblets and fungiform taste buds. Goblets cells are implicated in the lingual $\mathrm{V}$ between the anterior and posterior parts of the tongue. Foliated papillae are present on the posterior and lateral faces, they are formed of lymphoid tissues.

A covering epithelium is separated from the connective tissue by a basement membrane (Fig. $1,2)$. The outer compartment appears as a stratified, squamous epithelium. It is either ortho- or para-keratinized (stratum corneum with nucleated cells) located over the granular layer (stratum granulosum) (Fig. 3-5). Non-keratinized mucous membrane do not have a stratum corneum or granular or granulosum (Fig. 5-7).

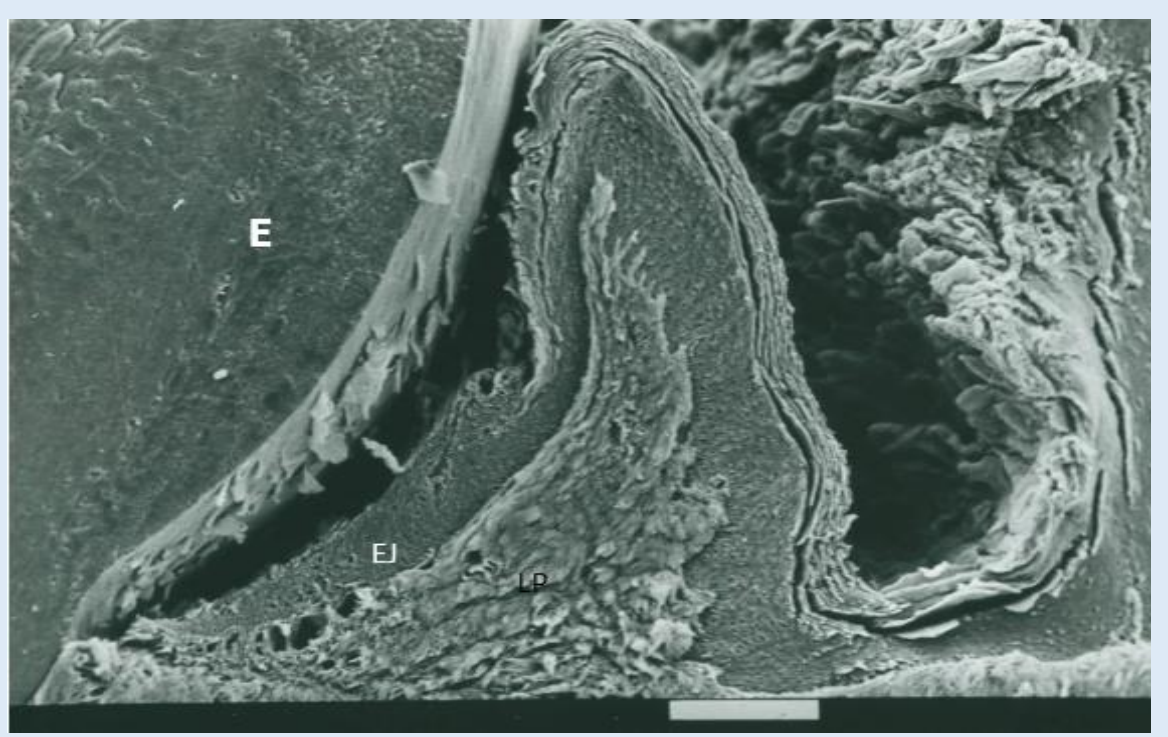

Figure 1: SEM section through gingiva. EJ: Enamel Junction E: Enamel

Goldberg M | Volume 1; Issue 1 (2020) | JDHOR-1(1)-002 | Review Article

Citation: Goldberg M. The Oral Mucosa: Subtypes of Epidermolysis Bullosa. J Dental Health Oral Res. 2020;1(1):1-10. 


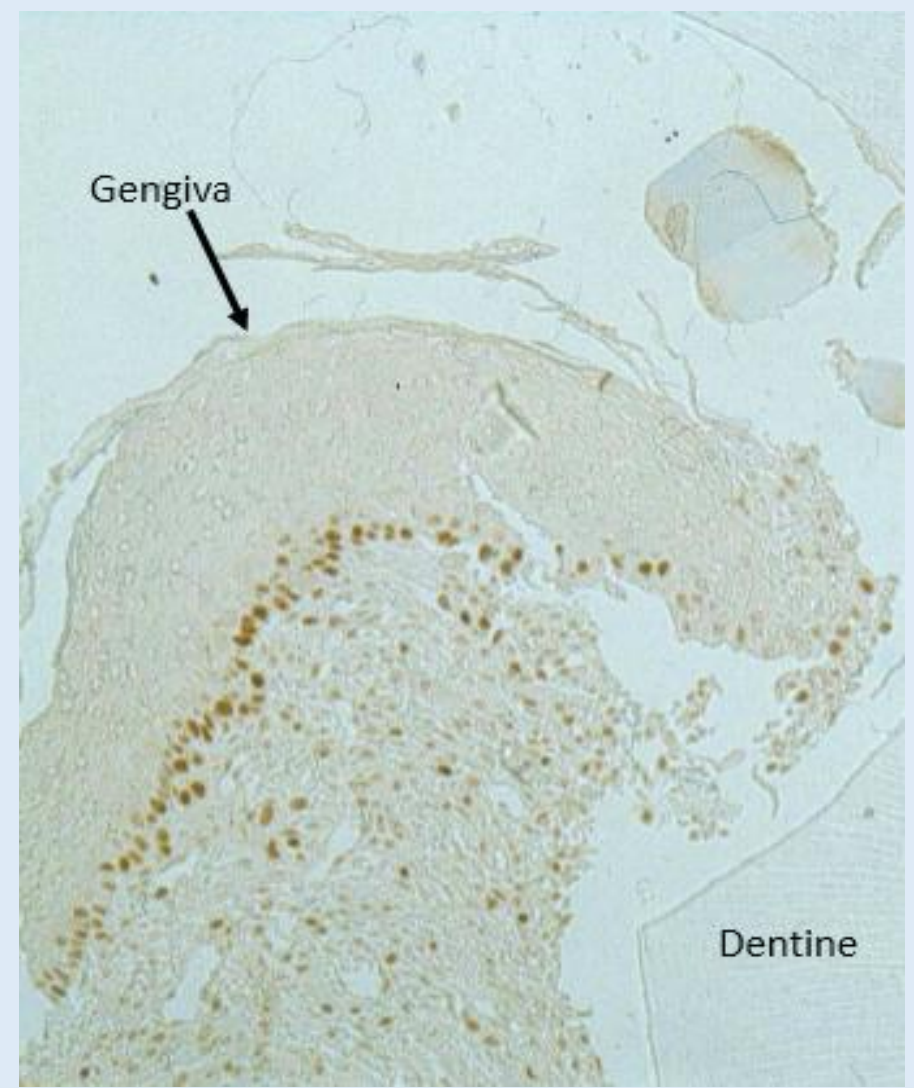

Figure 2: PCNA immunostaining of the gingiva. The proliferation marker label is seen only in the stratum basal.
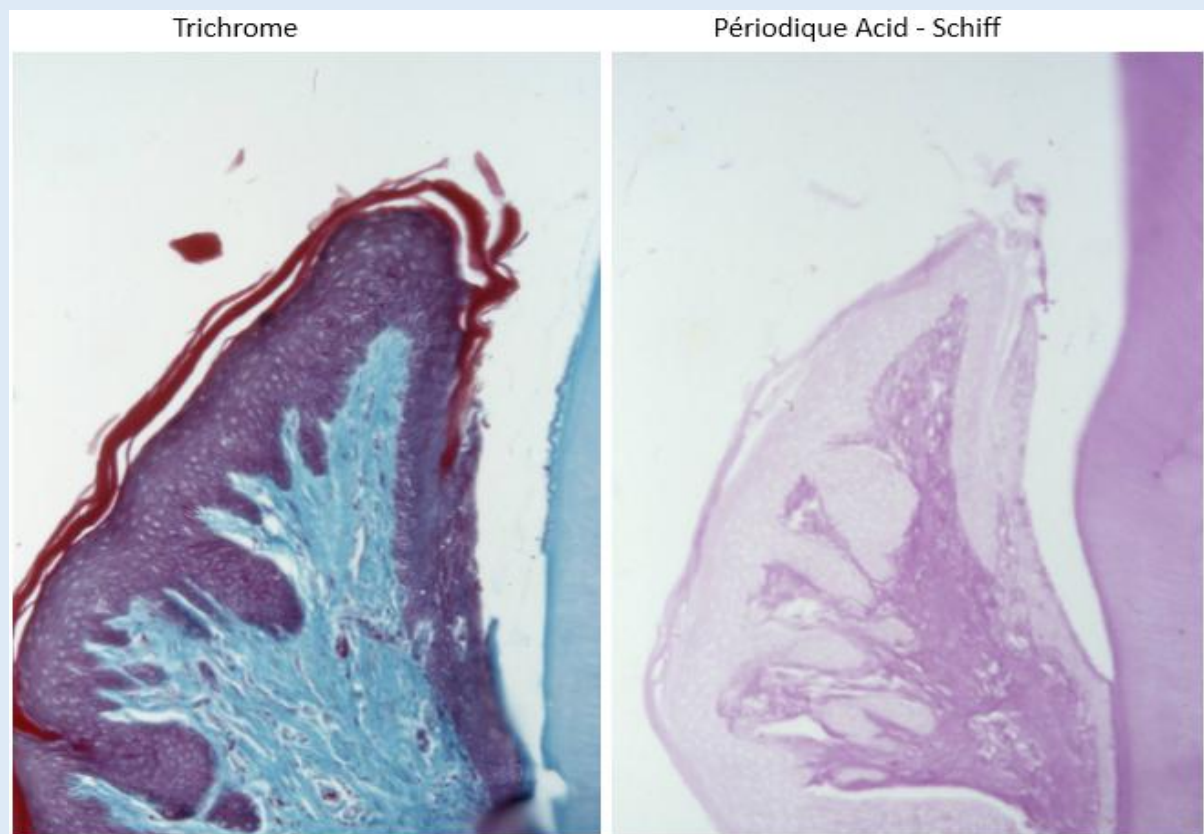

Figure 3: Trichrome allows to see the keratinized outer layer, epithelium in violet and the lamina propria in blue. Periodic acid-Schiff is pale pink in the epithelial part, and pink in the lamina propria (collagen staining).

Goldberg M | Volume 1; Issue 1 (2020) | JDHOR-1(1)-002 | Review Article

Citation: Goldberg M. The Oral Mucosa: Subtypes of Epidermolysis Bullosa. J Dental Health Oral Res. 2020;1(1):1-10. 


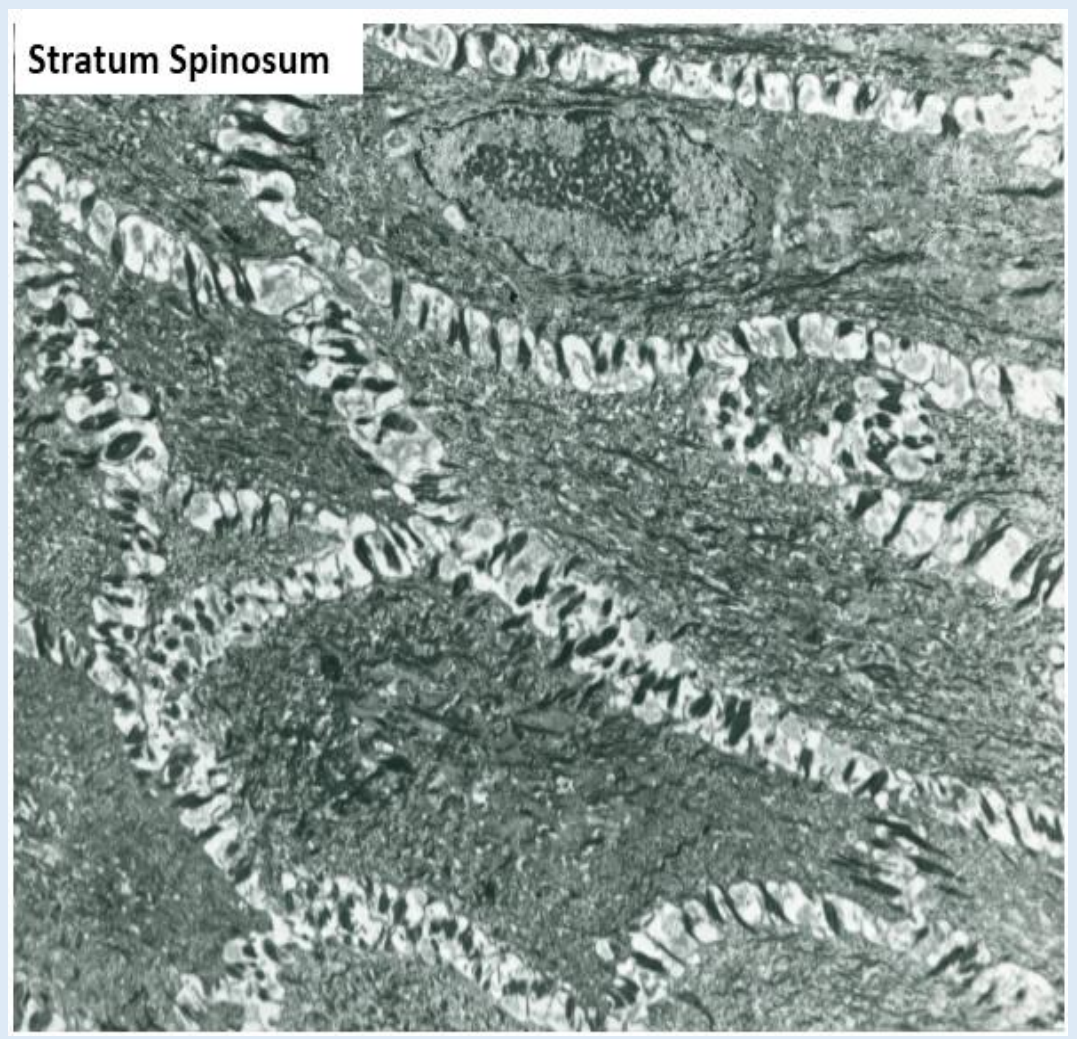

Figure 4: Epithelial stratum spinosum TEM ultrathin section.

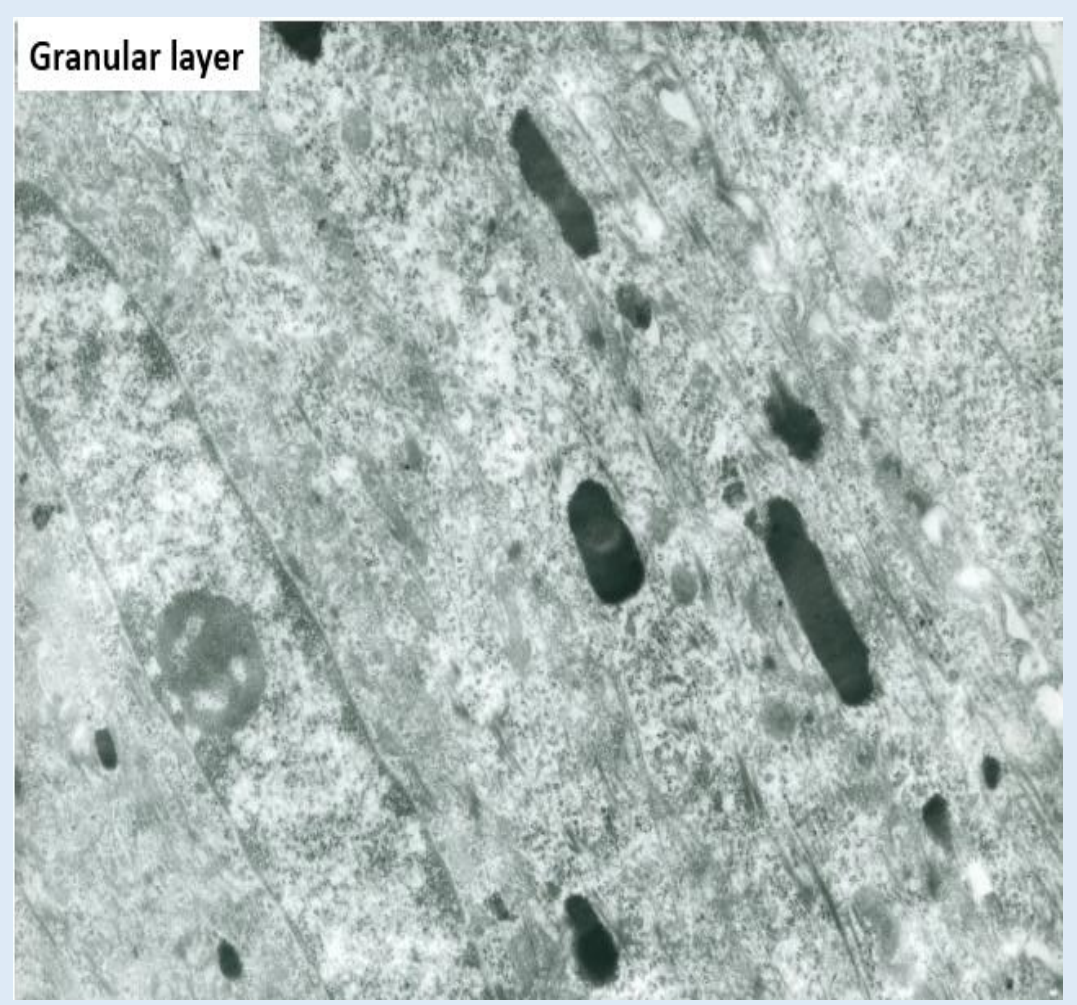

Figure 5: Stratum granulosum TEM ultrathin section.

Goldberg M | Volume 1; Issue 1 (2020) | JDHOR-1(1)-002 | Review Article

Citation: Goldberg M. The Oral Mucosa: Subtypes of Epidermolysis Bullosa. J Dental Health Oral Res. 2020;1(1):1-10. 


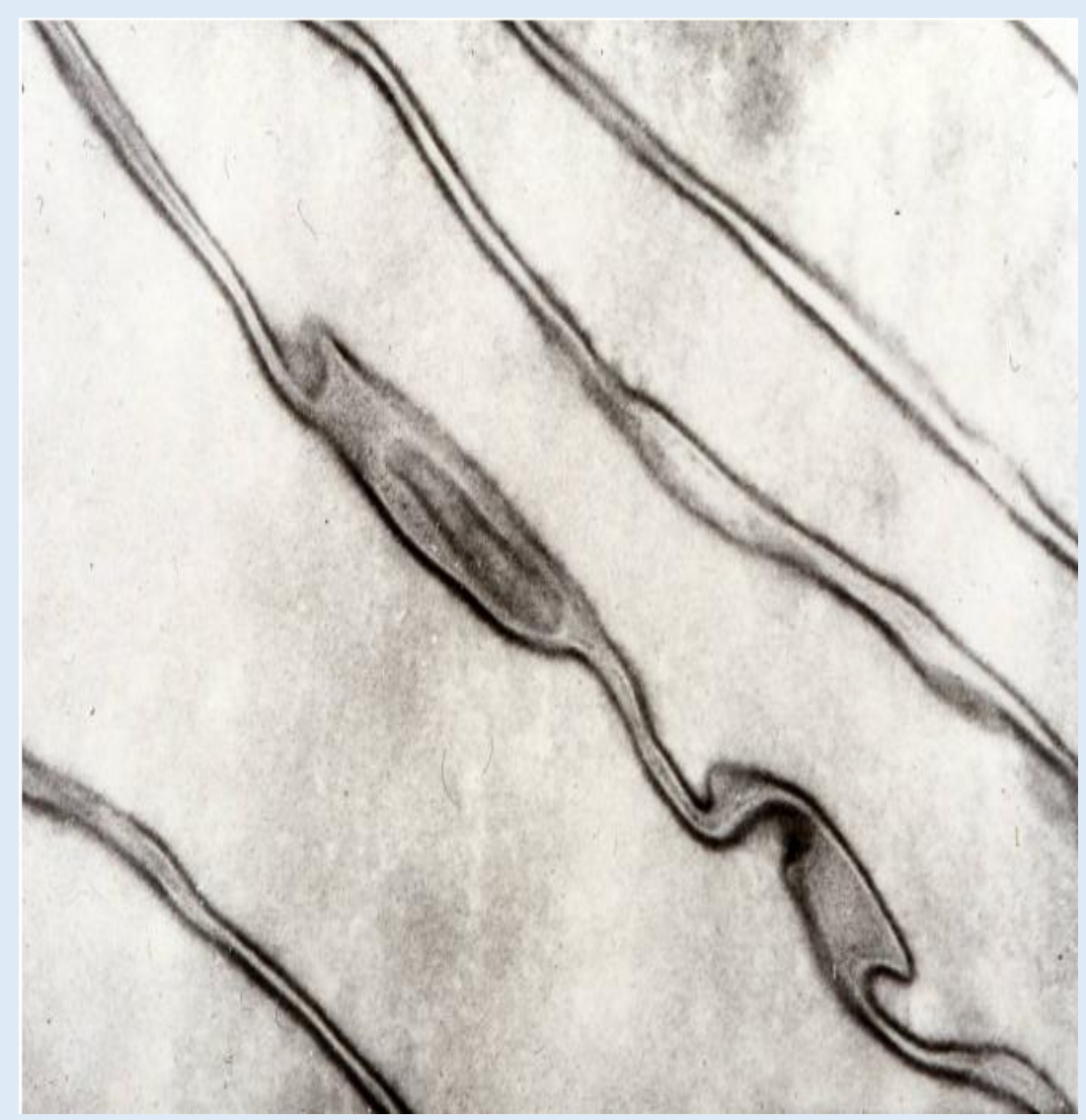

Figure 6: Keratinized layer TEM section.

The first epithelial cell layer is the stratum basale, in contact with the basement membrane (Fig.

2). The basal cells are formed by:

1) Stem /Progenitor Cells

2) Transient Amplifying Cells

3) Post-Mitotic Differentiating Cells

The layer that comes next is the stratum spinosum (Fig. 4), consisting of large polyhedral cells, joined by cells junctions giving to the cells a spinous appearance. The cells become flat, loaded with keratohyaline granules (granular keratinocytes), (Fig. 5).

This layer becomes gradually incorporated in the stratum granulosum (Fig. 5). The transition into stratum corneum (the outer keratinized layer) is characterized by the fact that the epithelial cells become flat, closely apposed to one another. The nuclei as well as cytoplasmic organelles disappear. The turnover of the oral mucosa epithelium is around 25 days, whereas in the skin the turnover time is 50 to 75 days. 
The basement membrane serves as an attachment to keratinocytes inserted by hemidesmosomes. A finger-like papillae contribute to the association with epithelial ridges on it's under face.

The lamina lucida is oriented toward the epithelium. The lamina densa is a filamentous middle layer and the lamina fibro-reticularis anchor the lamina densa and the underlying fibers to the chorion (Fig. 8,9).

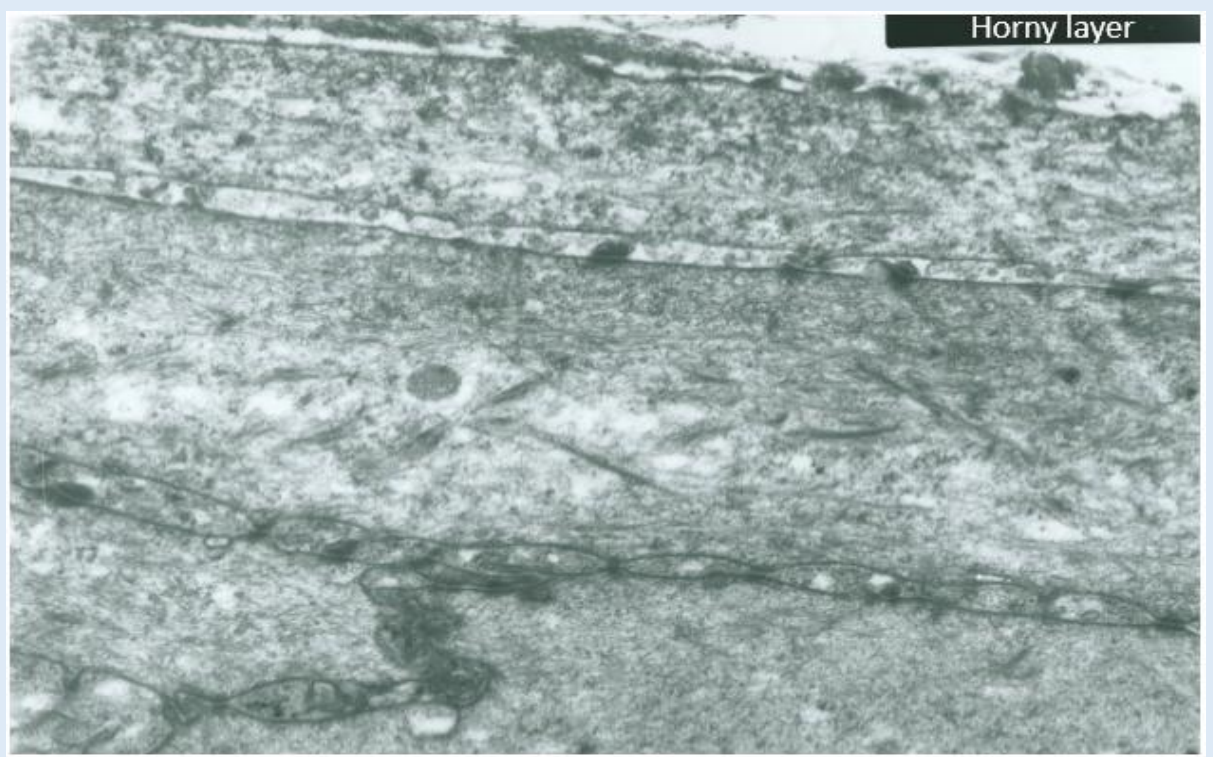

Figure 7: Keratinized (Horny layer) ultrathin section.
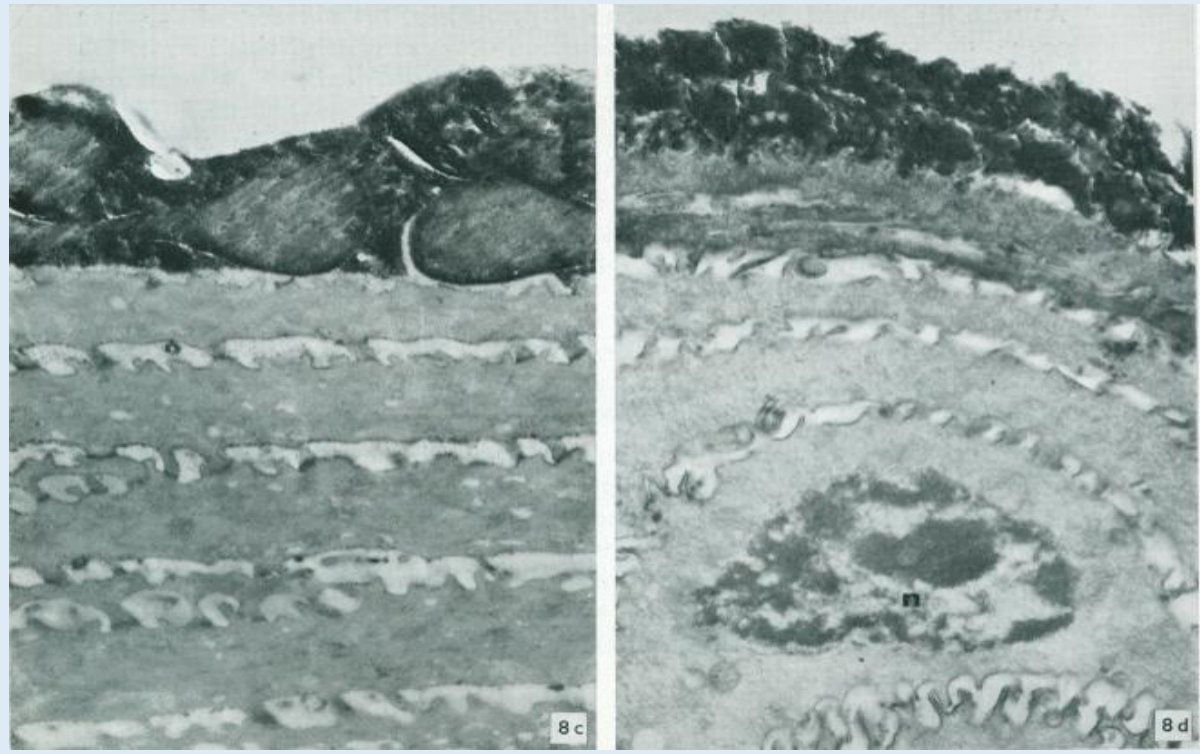

Figure 8: Permeability to horseradish peroxidase is limited to one outer layer (orthokeratinized) and to more epithelial layers (parakeratinized layer) with a nucleated cell ( $n=$ nucleus).

Goldberg M | Volume 1; Issue 1 (2020) | JDHOR-1(1)-002 | Review Article

Citation: Goldberg M. The Oral Mucosa: Subtypes of Epidermolysis Bullosa. J Dental Health Oral Res. 2020;1(1):1-10. 


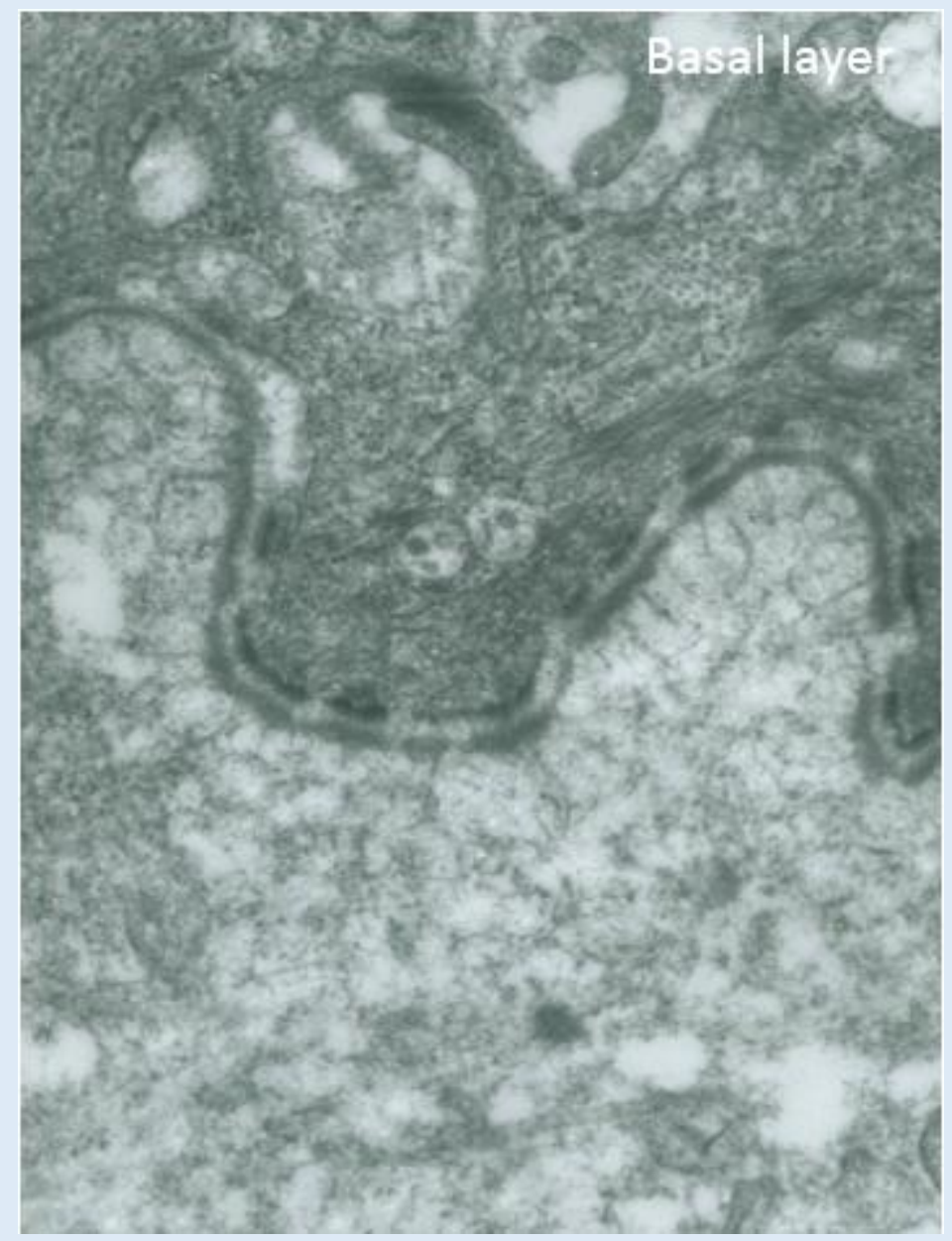

Figure 9: TEM section. The epithelial basal layer is seen in the upper part of the figure. The basement membrane is formed by a lamina lucida and a lamina densa. In the lower part of the figure, the papillary layer of the lamina propria is filled by collagen fibres.

The molecules implicated in the composition and structure of the basement membrane are: Type IV collagen forming a polygonal collagen type IV network, and the cell binding proteins associating the B1, B2, A chains of laminin and their isoforms, nidogen /entactin forming a stable complex with laminin, BM-40 (SPARC, osteonectin), and heparan sulfate proteoglycan [2]. In the stroma, anchoring fibers were identified as type VII collagen. In addition proteases were involved in many extracellular matrix proteins turnover (collagenases (MMP-2, MMP$9)$, growth factors and other non-catalytic regulatory proteins $[3,4]$.

The lamina propria is a component of the mucosal connective tissue. It consists of two compartments: the papillary and reticular layers (Fig. 3). The superficial papillary zone (the mucosa of the lamina propria) is tightly packed, whereas the reticular layer (submucosa of the lamina propria) constitutes a deep area of dense connective tissue (reticular layer). Accessory salivary glands are found in the submucosa of the lamina propria. The gingival connecting 
tissue includes collagen fibers, fibroblasts, vascular elements, and nerves. The masticatory mucosa is directly attached to the periostum of the underlying bone (Fig. 1,3).

The lamina propria is composed of a network of type I collagen, mixed with type III collagen, elastic fibers, glycosaminoglycans, proteoglycans, glycoproteins (fibronectin), Epidermal Growth Factor (EGF), Interleukin-1, colony-stimulating factor-1, monocyte chemotactic protein-1, metalloproteinases (MMP-9, MMP-2, and MMP-8), and mast cells (producing MMP-1 in the lamina propria). Osteoblasts and osteocytes are present near the bone. Occasional osteoclasts are found along the bone surface.

The histology and composition of the different zones of the oral mucosa play crucial roles in the development of oral pathologies, namely epidermolysis bullosa. Located at the epithelioconnective border, the blisters characterizing the different forms of epidermolysis occur either in the epithelial layer, basement membrane, or mucosa papillary zone or the oral submucosa (Fig. 6).

\section{The Different Forms of Epidermolysis Bullosa of the Oral Mucosa}

The following four subtypes of EB have been identified [5]. If the blister formation occurs within the epidermal layer, it is called epidermolysis bullosa simplex. When the blisters are located along the basement membrane it is known as junctional epidermolysis bullosa (JEB). Blister between the two different dermal layers is called the dystrophic form.

Epidermolysis Bullosa Simplex (EBS) has an autosomal recessive pattern are three major subtype of the basal epidermal keratins, the blistering being confined to the epidermis. This pathology was further separated into suprabasal and basal (Dowling Meara) subgroups including lethal acantolytic EB, plakophilin deficiency and EBS superficialis. Mild to severe forms with blistering is seen in the skin, but also in the mouth. Histologic cleavage occurs within the epidermis. Blisters develop within the mid-portion or junction (lamina lucida) of the basement membrane. Genetic disorders are also seen for the basal keratins genes (KRT5, KRT14) and for EBS with Mottled Pigmentation (MP) [6]. The prevalence of EBS is 19/100,000.

Junctional Epidermolysis Bullosa (JEB), in 1935, Hertlitz described a lethal form of epidermolysis bullosa, different from the simplex and dystrophic types (Herlitz-Pearson type). Another name was also given to this disease (Herlitz JEB, LAMB3-related). The number of cases encountered in this disease is 1-to 9 children/1,000 000. Two forms have been identified:

(1) A lethal form with a mutation reducing the labelling of laminin 5

(2) A non-Herlitz EB

The hemidesmosome-associated protein BP230 and play a role for the ectodomain in tumor development and invasion.

Goldberg M | Volume 1; Issue 1 (2020) | JDHOR-1(1)-002 | Review Article

Citation: Goldberg M. The Oral Mucosa: Subtypes of Epidermolysis Bullosa. J Dental Health Oral

Res. 2020;1(1):1-10.

DOI: http://dx.doi.org/10.46889/JDHOR.2020.1102 
Enamel is pitted. The most severe form is the JEB-Herlitz. This form displays ankyloglossia. Dystrophic Epidermolysis Bullos (DEB), is due to mutations in the gene encoding type VII collagen. The overall prevalence ranges from 1 to $8 / 1000000$ newborns. This pathology is due to mutations in the COL7A1 gene encoding the collagen type VII protein. Dominant or recessive DEB is a genetic defect, involving anchoring fibrils, forming a structural link in the dermis. The tongue forms severe ankyloglossia, and is closely associated with an atrophic tongue.

Kindler syndrome is the $4^{\text {th }}$ major type of EB. Besides simplex, junctional and dystrophic forms, this syndrome is characterized by skin fragility and blistering at birth. The prevalence is unknown. It results by a loss-of-function in the kindling-1 gene (FERMT1) [7-11].

Epidermolysis Bullosa Acquisita (EBA) is an autoimmune blistering disorder caused by autoantibody production. Five clinical presentations of EBA have been identified:

(i) Classic

(ii) Bullous penphigoid-like type EBA

(iii) Mucous Membrane Pemphigoid-like (MMP)-like

(iv) Brunstig-Perry pemphigoid-like

(v) Linear IgA Bullous Dermatosis (LAD)-like type EBA

\section{Conclusion and Treatment}

EB is an inherited genetic disease. Mutation in the COL7A1 have a consequence in making a protein (KRT 14) used to assemble type VII collagen. The proteins implicated in this construction were G2043R, G2775S, G1673R, G1338V and G2719A. EB17, with combined R2791W and G2210V variants, had a DDEB-Pasini phenotype. The life expectancy of children with EB is poor and most don't survive post the first year of life. Many die before they are 5 years old. Few children with EB live into adulthood. Most adults die before they are 30 years old.

There is no treatment that can control the etiology, but they can reduce the symptoms. They don't avoid skin damage, but improve the quality of life, reducing infections and malnutrition.

Blisters inside the mouth make cleaning the teeth difficult. Gum disease and cavities are common in people who have EB. Patients may need to see a dentist who has experience in treating EB. Appointments every 3 months are necessary. The "artificial nipple" includes soft food, not served too hot. It could be necessary to use milk-based drinks to reach the adequate level of proteins and calories. It may be necessary to have fibre supplement to reduce predisposing factor implicated in dental caries. Fluoride application may also contribute to control dental caries. 


\section{References}

1. Liu J, Mao JJ, Chen L. Epithelial-mesenchymal interactions as a working concept for oral mucosa regeneration. Tissue Engineering Part B: Reviews. 2011;17(1):25-31.

2. Timpl R. Structure and biological activity of basement membrane proteins. Eur J Biochem. 1989;180:487-502.

3. Listgarten MA. Normal development, structure, physiology and repair of gingival epithelium. Oral Sci Reviews. 1972;1:3-67.

4. Leblond CP, Inoue S. Structure, composition, and assembly of basement membrane. Am J Anat. 1989;185(4):367-90.

5. Fine JD, Bruckner-Tuderman L, Eady RA, Bauer EA, Bauer JW, Has C, et al. Inherited epidermolysis bullosa: updated recommendations on diagnosis and classification. J Am Acad Dermatol. 2014;70(6):1103-26.

6. Uttam J, Hutton E, Coulombe PA, Anton-Lamprecht I, Yu QC, Gedde-Dahl T, et al. The genetic basis of epidermolysis bullosa simplex with mottled pigmentation. Proc Natl Acad Sc. 1996;93(17):9079-84.

7. Tidman MJ, Eady RA. Hemidesmosome heterogeneity in junctional epidermolysis bullosa revealed by morphometric analysis. J Invest Dermatol. 1986;86(1):51-6.

8. Bruckner-Tuderman L, Höpfner B, Hammami-Hauasli N. Biology of anchoring fibrils: lessons from dystrophic epidermolysis bullosa. Matrix Biol. 1999;18(1):43-54.

9. Dang N, Klingberg S, Marr P, Murrell DF. Review of collagen VII sequence variants found in Australasian patients with dystrophic epidermolysis bullosa reveals nine novel COL7A1 variants. J Derm Sci. 2007;46(3):169-78.

10. Scheidt L, Sanabe ME, Diniz MB. Oral manifestations and dental management of epidermolysis bullosa simplex. Int J Clin Pediatr Dent. 2015;8(3):239-41.

11. Regina Kummer T, Cristhiane Müller Nagano H, Schaefer Tavares S, Zimmermann Dos Santos B, Miranda C. Oral manifestations and challenges in dental treatment of epidermolysis bullosa dystrophica. J Dent Child. 2013;80(2):97-100. 